\section{Journal Club}

\author{
PREPRINT WATCH
}

SEEING SARS-COV-2 VARIANTS THROUGH THE EYES OF T CELLS

The recent emergence of new SARS-CoV-2 variants of concern, including the B.1.1.7 variant in the UK, B.1.351 variant in South Africa, P.1 variant in Brazil and B.1.427 variant in California, USA, has important implications for future responses to the pandemic. Whereas the effects of mutations in the viral spike $(\mathrm{S})$ protein on antibody binding and neutralization have been addressed in several reports, the impact of SARS-CoV-2 variant mutations on T cell reactivity remains poorly understood.

In this non-peer-reviewed preprint, Tarke et al. applied an integrated approach to assess $T$ cell responses to SARS-CoV-2 variants from 11 COVID-19 convalescent individuals and 19 recipients of the Moderna (mRNA-1273) or Pfizer/BioNTech (BNT162b2) vaccines. Different methodologies were used to detect $T$ cells with a range of functionalities and specific cytokine activity in response to overlapping peptide pools spanning the $S$ protein of the original SARS-CoV-2 sequence and each of the corresponding variants.

Both $\mathrm{CD}^{+}{ }^{+}$and $\mathrm{CD} 8^{+} \mathrm{T}$ cells from COVID-19 convalescent donors were found to recognize the ancestral reference strain and the variant proteome-wide sequences with similar efficiency. In mRNA vaccine recipients also, $C D 4^{+}$ and $C D 8^{+} T$ cell responses to the ancestral and variant peptide pools were similar, with the exception of the B.1.351 variant, for which mildly decreased T cell reactivity to $S$ protein peptides was observed. Analysis of defined T cell epitopes showed that $93 \%$ of $C D 4^{+} \mathrm{T}$ cell epitopes and $97 \%$ of $C D 8{ }^{+} \mathrm{T}$ cell epitopes are conserved in the analysed variants. Single point mutations in the T cell epitopes were predicted to have no negative effect on HLA binding capacity, which provides a molecular basis for the marginal impact of the mutations on T cell responses in the study group.

Together, these findings suggest a negligible impact of the SARS-CoV-2 variant mutations on global $\mathrm{CD}^{+}$and $\mathrm{CD} 8^{+} \mathrm{T}$ cell responses in COVID-19 convalescent donors and COVID-19 mRNA vaccine recipients, and have important implications for the design of vaccines inducing broader immunity against variants of concern. The use of overlapping peptide pools in this study does not exclude the possibility that mutations described in the variants could interfere with antigen processing, thereby affecting $T$ cell activation and function. It remains to be determined whether $T$ cell responses following infection with circulating variants can efficiently cross-recognize the ancestral sequence in approved vaccines.

Aljawharah Alrubayyi and Dimitra Peppa OxMS Preprint Journal Club, Nuffield Department of Clinical Medicine, University of Oxford, Oxford, UK

凶e-mail: highlights@preprintclub.com

ORIGINAL ARTICLE Tarke, A. et al. Negligible impact of SARS-CoV-2 variants on $\mathrm{CD}^{+}$and $C D 8^{+} \mathrm{T}$ cell reactivity in COVID-19 exposed donors and vaccinees. Preprint at bioRxiv https://doi.org/10.1101/2021.02.27.433180 (2021) RELATED ARTICLE Alrubayyi, A. Oxford-Mount Sinai (OxMS) Preprint Journal Club. OxMS https://www.preprintclub.com/2021-mar-tarke (2021)

\title{
Mechanosurveillance of tumour metastasis
}

Immunosurveillance of cancer cells by cytotoxic lymphocytes depends on the receptor recognition of cellsurface ligands indicative of stress and/or transformation, resulting in the formation of a cytotoxic synapse. Several of these receptors are further activated by the mechanical forces exerted by the synapse (known as mechanotransduction), and indeed increased rigidity of the target surface correlates with increased lymphocyte activation. Given that tumour metastasis involves marked cytoskeletal remodelling, Tello-Lafoz et al. set out to investigate whether these biophysical changes also affect lymphocyte activation and killing.

Metastasis involves the activation of myocardin-related transcription factor A (MRTFA) and MRTFB by cancer cells. As expected, therefore, suppression of MRTF expression reduced lung colonization by melanoma and breast cancer cell lines in a mouse model of metastasis. Surprisingly, however, overexpression of MRTFB also reduced lung colonization. The metastatic activity of MRTF-overexpressing cells could be rescued by depletion of cytotoxic lymphocytes, which suggests that the metastatic potential conferred by MRTF activation might be partially restricted by the immune system.

Further studies showed that MRTF-overexpressing cancer cells are more susceptible to cytotoxicity, with synapse formation more likely to result in cancer cell death and increased speed of killing. MRTF-overexpressing cells induced stronger degranulation of cytotoxic lymphocytes than control cells, which is consistent with MRTF overexpression inducing stronger lymphocyte activation.

\section{Variant constraint by mRNA vaccines}

Several vaccines against SARS-CoV-2 are now licensed for use, but there is concern that they may be less effective against emerging variants of the virus. Stamatatos et al. now report that the mRNA vaccines designed against earlier variants of SARS-CoV-2 also elicit and boost levels of cross-neutralizing antibodies (nAbs) to newer B.1.351 variants, but are less potent against these variants.

The two mRNA vaccines that have received emergency use authorizations - BNT162b2 (Pfizer/BioNTech) and mRNA-1273 (Moderna) - encode a stabilized ectodomain version of the spike (S) protein from the Wuhan-Hu-1 variant of SARS-CoV-2 (isolated in 2019). These vaccines elicit nAbs to the virus and show more than $94 \%$ efficacy in preventing disease. However, emerging variants of SARS-CoV-2 contain $S$ protein mutations that could potentially evade the $\mathrm{nAb}$ responses induced by the vaccines. Of particular concern are variants that have emerged in the United Kingdom (B.1.1.7), South Africa (B.1.351) and Brazil (P.1) as they have mutations that may enhance transmissibility and disease severity, as well as vaccine evasion.

The authors collected sera from 15 individuals who had previously been

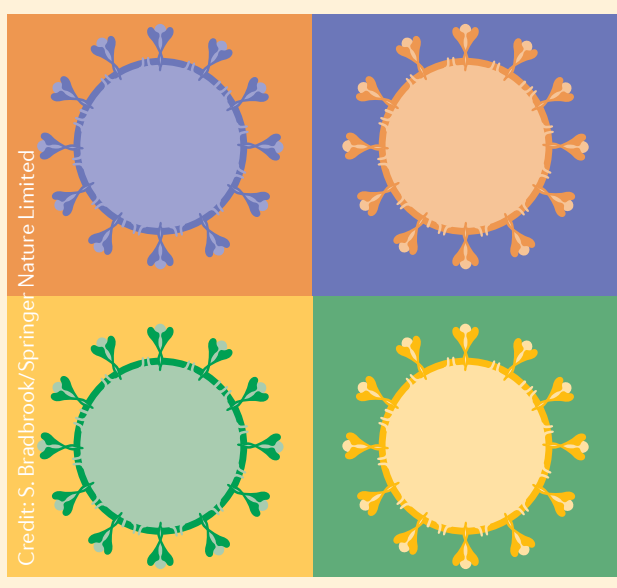

